\title{
Accuracy in Diagnosis of Acute Appendicitis by Comparing Serum C - reactive protein Measurements and Modified Alvarado Score
}

\author{
Dr Joel Mathew John ${ }^{1}$, Dr K.N. Rajan Babu ${ }^{2}$ \\ ${ }^{I}$ (Junior Consultant-General Surgery) \\ ${ }^{2}$ (Associate Professor, Dept. Of General Surgery, Pushpagiri Institute Of Medical Sciences And Research \\ Centre,Thiruvalla,Kerala,India)
}

\begin{abstract}
Acute appendicitis is perhaps the most common surgically correctable cause ofacute abdominal pain and its diagnosis remains difficult in many instances. Some ofthe signs and symptoms can be subtle to both the clinician and the patient and may notbe present in all the instances. Arriving at the correct diagnosis and decreasing therate of negative appendicectomy is essential. However a delay in diagnosis may allowprogression to perforation and significantly increase the morbidity and mortality.This study was a prospective study and comprised 60 patients admitted with suspected acute appendicitis and planned for Appendicectomy. The patients selected were older than 15 years, of either sex. The preoperative modified Alvarado score (MAS) was determined and C-reactive protein levels (CRP) were estimated. The results of the MAS and preoperative CRP levels were compared with the histopathology of the removed appendix.Among all the patients studied, 6, 29 and 25 patients had preoperative MAS of <5, 5-6, and 7-9 respectively. 42 patients had CRP level raised. 41 patients were histopathologically positive for appendicitis. 55\% and 88\% of patients with a MAS of 5-6 and 7-9 had appendicitis on histopathology respectively. We found $82.9 \%$ sensitivity and $57.9 \%$ specificity of CRP for the diagnosis of acute appendicitis. When combined with CRP, the sensitivity and specificity of MAS in patients with score of 5-6 was raised significantly from 55\% to 75\% and 45\% to 61.5\%. CRP was raised in all patients with MAS score of 7-9.Our study concluded that MAS in combination with the CRP levels is very helpful in the diagnosis of acute appendicitis especially in patients with modified Alvarado scores in the middle range, who are categorized as "equivocal" for appendicitis by the Alvarado score.
\end{abstract}

Keywords: Acute Appendicitis,Appendicectomy,C-Reactive Protein, Modified Alvarado Score.

\section{Introduction}

The appendix is a structure without apparent proven function, yet it has been afrequent cause of morbidity and mortality.Appendicitis, an infection of the appendix may occur for several reasons.However, the usual picture of appendicitis is often not classical, leaving in many cases a diagnostic problem.In patients with questionable findings, the aggressive surgical approach has been "when in doubt, take it out," and the price paid was the frequent removal of normal appendices. This negative laparotomy is associated with definite morbidity but the mortality rate is minimal compared to the lethal potential of appendiceal perforation and peritonitis[1].Diagnostic scoring systems have been developed in an attempt to improve the diagnostic accuracy of acute appendicitis. The most prominent of those scores is that developed by Alvarado[2].Subsequent prospective studies have suggested that theAlvarado score alone is inadequate as a diagnostic test $[3,4]$ The classic Alvarado score included left shift of neutrophil maturation (given a score of 1) yielding a total score of 10 but Kalan et al.[5]omitted this parameter which is not routinely available in many laboratories, and produced a modified score.An elevated level of C-reactive protein (CRP), an acute phase protein, is one of many downstream indicators of inflammation. In most diseases, the circulating value of CRP reflects ongoing inflammation and/or tissue damage much more accurately than do other laboratory parameters of the acutephase response. The CRP concentration is thus a very useful but a nonspecific biochemical marker of inflammation, measurement of which contributes importantly to (a) screening for organic disease, (b) monitoring of the response to treatment of inflammation and infection, and (c) detection of intercurrent infection in immune compromised individuals[6].The aim of this study was to determine the sensitivity and specificity of MAS separately and in combination with the CRP, to ensure preoperative diagnostic accuracy of appendicitis in patients with suspected acute appendicitis. 


\section{Material's And Method}

A prospective study was conducted in the Department of General Surgery of Pushpagiri Institute of Medical sciences and Research Centre, Thiruvalla. It included 60 patients from November 2013 to July 2015 suspected of suffering from acute appendicitis warranting emergency surgery. The scoring system used for clinical evaluation is Modified Alvarado score with a maximum score of 9. All patients included in this study were older than 15 years of age and presented with acute right iliac fossa pain. A thorough elicitation of the history and physical examination were carried out including all the components of the Modified Alvarado score and preoperative estimation of Serum CRP. These patients were subjected tosurgery and the appendix specimen was sent for histopathological evaluation (referencestandard).The performance of the Modified Alvarado score and CRP in comparison withHistopathology was studied. A negative appendicectomy was considered if the histopathology report stated "normal appendix" or "appendix with lymphoid hyperplasia"The CRP was measured using the nephelometry method of estimation.CRP of up to $1 \mathrm{mg} / \mathrm{dl}$ was considered the upper limit of normal standard. Decision to operate was not affected by the preoperative CRP levels. The CRP levels was not revealed to the operating surgeon preoperatively.

\section{Modified Alvarado Score:}

Symptoms M = Migratory right iliac pain 1

A = Anorexia-

$\mathrm{N}=$ Nausea/vomiting

1

1

Signs $\mathrm{T}=$ Tenderness right lower quadrant 2

$\mathrm{R}=$ Rebound tenderness right iliac fossa

$\mathrm{E}=$ Elevated temperature[ $>37.3] \quad 1$

Investigation $\quad \mathrm{L}=$ Leucocytosis $[>10,500]$

Total score-

The estimated score was not revealed to the operating surgeon preoperatively.Therespective sensitivity and specificity of MAS, CRP and MAS with CRP was calculatedaccordingly with positive and negative histopathology for Appendicitis. On the basis ofMAS, our study grouped the patients as "unlikely" (score $<5$ ), "possible" (score 5-6) and"probable" appendicitis (score 7-9).

\section{Results}

The study was conducted on 60 selected patients of age group $>15$ years andeither sex with the age range of $17-71$ years. The mean age and SD of the patients was 28.35 years \pm 13.1 . There were 25 males and 35 females.

Table 1: Sex Distribution of Patients

\begin{tabular}{l|c|c|c} 
Sex & Positive & Negative & Total \\
\hline Male & 19 & 6 & 25 \\
\hline Female & 22 & 13 & 35
\end{tabular}

Out of 25 male patients, 19 were positive for appendicitis. Similarly out of 35 femalepatients, 22 were positive for appendicitis.

Table 2: Age vs Sex Correlation amongst Patients

\begin{tabular}{l|c|c|c} 
Sex & Age : Mean \pm SD & t & p value \\
\hline Males & $27.3 \pm 13.7$ & \multirow{2}{*}{0.532} & 0.60 \\
\hline Females & $29.1 \pm 12.8$ & & \\
\hline
\end{tabular}

The preoperative Modified Alvarado score of all the patients included in the study was determined. On the basis of this scoring, there were 6 patients with score of <5(Unlikely), 29 with a score of 5-6(Possible), and 25 with a score of 7-9 (Probable). 


\begin{tabular}{c|c|c|c|}
\multicolumn{5}{c|}{ Table 3 : MAS vs Histopathology } \\
\hline MAS & Positive Histopathology & Negative Histopathology & Total \\
\hline Unlikely (<5) & 3 & 3 & 6 \\
\hline Possible (5-6) & 16 & 13 & 29 \\
\hline Probable(7-9) & 22 & 3 & 25
\end{tabular}

Out of 60 patients, 6 patients had a MAS of $<5,29$ patients had a MAS of 5-6 and 25 patients had a MAS of 7-9.My study showed positive appendicitis in 50\%, 55\% and 88\% of the "unlikely,"“possible," and "probable" groups respectively.

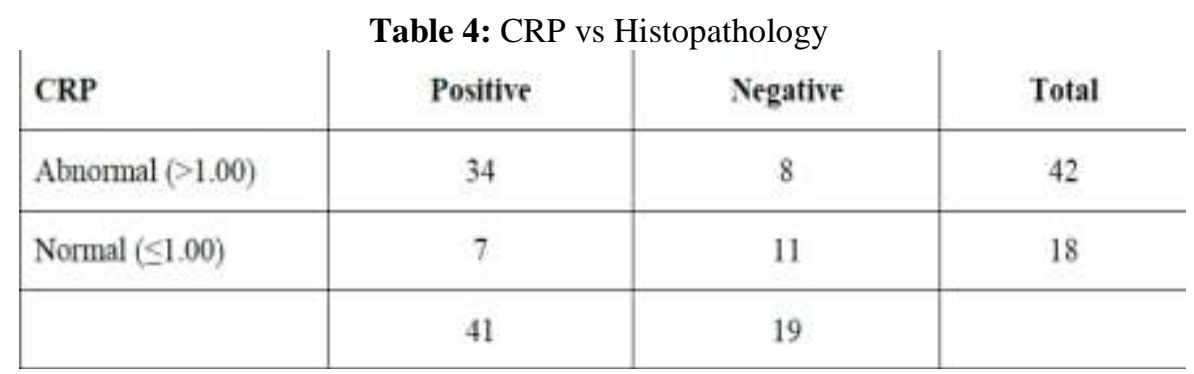

Out of 60 patients, 42 patients had raised levels of CRP and 18 patients had anormal level of CRP. 34 patients with raised CRP had positive histopathology showingappendicitis. Only 7 patients had positive histopathology with normal CRPSensitivity, specificity, PPV and NPV of CRP in my study was 82.9 \%, 57.9 $\%, 80.9 \%$ and $61.1 \%$ respectively.

Table 5: CRP vs Histopathology in 'Unlikely' group

\begin{tabular}{|l|c|c|}
\hline Unlikely(<5) & Positive & Negative \\
\hline Raised CRP & Nil & Nil \\
\hline Normal CRP & 3 & 3 \\
\hline
\end{tabular}

Table 6: CRP vs Histopathology in 'Possible' group

\begin{tabular}{|l|c|c|} 
Possible (5-6) & Positive & Negative \\
\hline Raised CRP & 12 & 5 \\
\hline Normal CRP & 4 & 8 \\
\hline
\end{tabular}

All the 6 patients with MAS $<5$ had normal CRP, and 3 patients in this group hadpositive histopathology. 12 out of 17 patients with MAS 5-6 and raised CRP had positive histopathology of appendicitis. When MAS and CRP levels were combined, the „Possible" group obtained a sensitivity of $75 \%$ and a specificity of $61.5 \%$. The PPV and NPV was $70.5 \%$ and $66.6 \%$ respectively.

Table 7: CRP vs Histopathology in 'Probable' group

\begin{tabular}{|l|c|c|}
\multicolumn{3}{|c}{ Table 7: CRP vs Histopathology in 'Probable' group } \\
Probable (7-9) & Positive & Negative \\
\hline Raised CRP & 22 & 3 \\
\hline Normal CRP & Nil & Nil
\end{tabular}

22 out 25 patients with MAS 7-9 had positive histopathology of appendicitis. There were no patients who had normal CRP in this group. 


\section{Discussion}

Acute appendicitis is one of the most common causes of acute abdominal pain in the Western world. The mainstay treatment of which continues to be the surgical removal of the appendix. However, since the symptomatology and the clinical picture can overlap with other inflammatory and non inflammatory conditions of the digestive, reproductive or the genitourinary system; and also with the incidence of negative appendicectomy of about $20 \%$; several newer laboratory and imaging techniques have been attempted to supplement the surgeons clinical impression. The estimation of $\mathrm{C}$ - reactive protein (CRP) as an adjunct with Modified Alvarado score has been evaluated in this study. Several studies have showed favourable results for this inflammatory marker. Gurleyik et al hasreported the sensitivity, specificity and accuracy of serum CRP measurements in Appendicitis to be 93.5, 80, and 91 percent, respectively. The mean range of CRP in non perforated and perforated appendicitis was 3.38 and $1.285 \mathrm{mg} / \mathrm{dl}$ respectively [7]. When the threshold of CRP was raised and also when it was combined with leucocytosis as a marker, the diagnostic value of CRP was found to be better. ShozoYokoyamaet al reported a cut off value of CRP of $4.95 \mathrm{mg} / \mathrm{dl}$ as the onlymarker for surgical indication of appendicitis versus conservative medical management [8].Kumar et al studied that CRP had a sensitivity of $94.4 \%$ and a positive predictive valueof $95.5 \%$. When CRP reactivity and leucocytosis were combined, the sensitivity, specificity, PPV and NPV were $85 \%, 100 \%, 100 \%$ and $81 \%$ respectively. When the threshold for CRP reactivity was raised to $2.4 \mathrm{mg} / \mathrm{dl}$, the sensitivity, specificity, PPV and NPV are $42 \%, 100 \%$, $100 \%$ and $16 \%$ respectively. Hence, concluding CRP estimation to be a good 'rule-in' test and not-so-good 'ruleout' test to diagnose acute appendicitis [9].However, the fact that these raised inflammatory markers may indicate a pathological inflammatory process and not that of a specific diagnosis of acute appendicitis has raised concerns. Jangjoo et al supported this with an estimated sensitivity and specificity of 59\% and $68 \%$ respectively [10].AnshumanSengupta et al contraindicated the findings of other studies in that the presence of raised inflammatory markers is not a good discriminator for patients with acute appendicitis, even when both leucocytosis and CRP levels are raised. They have in fact noted a very important finding that there is a $100 \%$ negative predictive value for acute appendicitis if both leucocyte counts and CRP are normal, i.e. no patients with both values within the normal range had acute appendicitis [11].

In our study, all the patients included were operated and decision to operate was not prejudiced by the preoperative modified Alvarado scoring or the level of CRP. On the basis of MAS, Gyomber D and Luck, grouped the patients as "unlikely" (score <5), "possible" (score 5-6) and "probable" appendicitis (score 7-9). Comparing histological findings to the modified Alvarado score, positive appendicitis was found in $38 \%, 73 \%$, and 93\% of the "unlikely," "possible," and "probable" groups respectively [12].Our study showed positive appendicitis in 50\%,55\% and 88\% of the "unlikely," "possible," and "probable" groups respectively. Out of 19 patients with negative appendix, 8 had a raised value of CRP and 11 patients had CRP levels within normal range.Sensitivity and specificity of CRP in this study was $82.9 \%$, and $57.9 \%$ respectively.Here, 19 of 60 patients with negative appendectomy had CRP levels within the normal range. If preoperative serum levels of CRP were to be believed, 19 negative laparotomies would have been avoided. However when MAS and CRP levels were combined, the sensitivity of the ,possible group rose from 55\% to $75 \%$ and specificity rose from $45 \%$ to $61.5 \%$. Just as Chan, et al. found that the Alvarado score was most accurate only at the two extremes of the score [13] the present study showed that the best sensitivity and specificity scores were obtained at MAS <5 and 7-9. Even though the rise in sensitivity of the ,possible ee group isn't as high as reported by Shafi ,et al (sensitivity rise from $58 \%$ to $93 \%$.). there is a significant improvement compared to evaluation by MAS alone. This study also shows a significant rise in specificity compared to that reported by Shafi ,et al (specificity rise from $13.5 \%$ to $23.5 \%$.).[14], which may imply that thismethod is a good, ,ruling out test in the possible group.

\section{Conclusion}

Acute appendicitis is one the most common surgically correctable cause of acute abdominal pain in the West and in India. Its diagnosis can be difficult in many instances.Some of the signs and symptoms can be subtle to both the clinician and the patient and may not be present in all the instances. Arriving at the correct diagnosis at the correct time is essential; however a delay in diagnosis may allow progression to perforation and significantly increase the morbidity and mortality. At the same time, subjecting a patient to a negative appendicectomy or negative laparotomy can also increase unnecessary morbidity. In spite of various advances in laboratory and radiological investigations to diagnose appendicitis, the clinical impression of the surgeon plays a vital role. Our study found that the diagnostic accuracy of acute appendicitis on the basis of clinical examination or MAS was sensitive, but does not contribute significantly to the diagnosis in patients with equivocal clinical findings. CRP levels were found to be highly sensitive and specific for the diagnosis of acute appendicitis. The sensitivity and specificity was increased when CRP levels were combined with MAS. When CRP levels were within the normal limits and the scores were low, acute appendicitis was very unlikely. We found MAS to be most accurate at the extreme of the scores. When CRP was also incorporated in the patients with the middle 
score of 5-6 (equivocal for appendicitis), the sensitivity and specificity rose significantly. Our study showed that in patients with high MAS and raised CRP levels, the probability of acute appendicitis was significantly increased. We believe that both MAS and CRP levels should be estimated in all patients who come to hospital with complaints of pain in the right iliac fossa and have equivocal clinical findings. These are not overtly expensive to the patient, are non-invasive, and do not require any sophisticated equipments or technical expertise. Moreover, the results of this investigation can be obtained promptly allowing the surgeon to establish diagnosis immediately and thereby avoiding unwanted explorations and preventing complications (perforation, abscess).We conclude that MAS in combination with the CRP levels is very helpful in the diagnosis of acute appendicitis especially in patients with Modified Alvarado scores in the middle range, who are categorized as "equivocal" for appendicitis by the Alvarado score.

\section{Acknowledgement}

I remember with gratitude the efforts taken by Mrs. Nisha Kurien, AssistantProfessor, Biostatistics to help me through with valuable suggestions and correctionsduringthe entire course of my thesis. Last, but not the least, I convey my heartfelt gratitude to allthe patients without whose cooperation, this study would have been incomplete.

\section{References}

[1]. Teicher IR, Landa B, Cohen M, Kabnick LS, Wise L. Scoring system to aid indiagnosis of acute appendicitis. Ann Surg 1983;198:753-9.

[2]. Zinner MJ, Ashley SW. Maingot's Abdominal Operations. 11 th ed. McGraw Hill;2007. p. 589-608

[3]. Ohmann C, Yang Q, Franke C. Diagnostic scores for acute appendicitis. Eur J Surg 1995;161:273-81.

[4]. Macklin CP, Radcliffe GS, Merei JM, Stringer MD. A prospective evaluation of themodified Alvarado scores for acute appendicitis in children. Ann R CollSurgEngl1997;79:2035.

[5]. Kalan M, Talbot D, Cunliffe WJ, Rich AJ. Evaluation of the modified Alvarado scorein the diagnosis of acute appendicitis: A rospective study. Ann R CollSurgEngl1994;76:418-9

[6]. Pepys MB, Hirschfield GM. C-reactive protein: A critical update. J Clin Invest2003;111:1805-12.

[7]. Gomez A, Wood M: Acute appendicitis during pregnancy. Am JSurg1979; 137:180.

[8]. Sauerland S, Lefering R, Neugebauer EA. Laparoscopic versus open surgery forsuspectedappendicitis. Cochrane Database Syst Rev. 2004 Oct 18. CD001546.

[9]. McBurney C. II. The Indications for Early Laparotomy in Appendicitis.Ann Surg.1891 Apr. 13(4):233-54.

[10]. Arnbjornsson E: Management of appendiceal abscess. CurrSurg; 1984; 41: 4-9.

[11]. Cariati A, Brignole E, Tonelli E, Filippi M, Guasone F, De Negri A. [Laparoscopic oropen appendectomy. Critical review of the literature and personal experience].GChir. 2001 Oct. 22(10):353-7.

[12]. Semm K. Endoscopic appendectomy.Endoscopy. 1983 Mar. 15(2):59-64.

[13]. Korndorffer JR Jr, Fellinger E, Reed W. SAGES guideline for laparoscopicappendectomy. SurgEndosc. 2010 Apr. 24(4):757-61

[14]. Esposito C, Borzi P, Valla JS, Mekki M, Nouri A, Becmeur F. Laparoscopic versusopen appendectomy in children: a retrospective comparative study of 2,332cases. World J Surg. 2007 Apr. 31(4):750-5. 